\begin{tabular}{|c|c|}
\hline Title & A pplications of the Lace Expansion to Statistical-Mechanical Models \\
\hline Author(s) & Sakai, A kira \\
\hline Citation & $\begin{array}{l}\text { Chapter: } 3.1,123-147 \\
\text { https://doi.org/10.1093/Acprof:oso } 9780199239252.003 .0006 \\
\text { A nalysis and stochastics of growth processes and interface models }\end{array}$ \\
\hline Issue Date & 2008 \\
\hline Doc URL & http:/hdl.handle.net/2115/57861 \\
\hline Rights & $\begin{array}{l}\text { "A pplications of the lace expansion to statistical-mechanical models" by "A nalysis and Stochastics of Growth Processes } \\
\text { and Interface Models" edited by Peter Mörters ...[et al.], 2008, reproduced by permission of Oxford University Press } \\
\text { http://doi.org/10.1093/acprof:0so/9780199239252.003.0006 }\end{array}$ \\
\hline Type & article (author version) \\
\hline File Information & 9780199239252.003.0006.pdf \\
\hline
\end{tabular}

Instructions for use 


\title{
Applications of the lace expansion to statistical-mechanical models
}

\author{
Akira Sakai*
}

August 31, 2007

\section{Introduction}

Synergetics is a common feature in interesting statistical-mechanical problems. One of the most important examples of synergetics is the emergence of a second-order phase transition and critical behavior. It is rich and still far from fully understood. The reason why it is so difficult is due to the increase to infinity of the number of strongly correlated variables in the vicinity of the critical point. For example, the Ising model, which is a model for magnets, exhibits critical behavior as the temperature $T$ comes closer to its critical value $T_{\mathrm{c}}$; the closer the temperature $T$ is to $T_{\mathrm{c}}$, the more spin variables cooperate with each other to attain the global magnetization. In this regime, neither standard probability theory for independent random variables nor naive perturbation techniques work. The lace expansion, which is the topic of this article, is currently one of the few approaches to rigorous investigation of critical behavior for various statistical-mechanical models. We summarize here some of the most intriguing lace-expansion results for selfavoiding walk (SAW), percolation and the Ising model. We also briefly explain the proof based on the latest version of bootstrapping argument in $[7,18,27]$ and (the first few stages of) the derivation of the lace expansion for those three models.

\subsection{Models}

Let $D$ be either the step distribution of the simple symmetric random walk (i.e., $D(x)=$ $\frac{1}{2 d} \mathbb{1}_{\{|x|=1\}}$, where $\mathbb{1}_{\{\ldots\}}$ is the indicator function) or the following spread-out probability distribution on $\mathbb{Z}^{d}$. Given $L \in[1, \infty)$, we let $h_{L}$ be a piecewise-continuous bounded probability distribution on $\mathbb{R}^{d}$ that respects the $\mathbb{Z}^{d}$-symmetry and satisfies $h_{L}(L u)>0$ for any unit vector $u \in \mathbb{Z}^{d}$ and $h_{L}(o)=0$ at the origin $o=(0, \ldots, 0)$. Then, we define

$$
D(x)=\frac{h_{L}(x / L)}{\sum_{y \in \mathbb{Z}^{d}} h_{L}(y / L)},
$$

where $x / L=\left(x_{1} / L, \ldots, x_{d} / L\right) \in \mathbb{Z}^{d} / L$. By the assumed bound on $h_{L}$, we have $D(o)=0$ and $D(u)>0$ for any unit vector $u \in \mathbb{Z}^{d}$. Moreover, by the Riemann-sum approximation,

\footnotetext{
${ }^{*}$ Creative Research Initiative "Sousei", Hokkaido University, Japan. sakai@cris.hokudai.ac.jp

${ }^{\dagger}$ Updated: April 4, 2008
} 
the denominator in (1.1) is $O\left(L^{d}\right)$, which plays a significant role in the analysis of the lace expansion.

We will use $D$ as a microscopic coupling of the models on $\mathbb{Z}^{d}$. We refer to a model defined by $D(x)=\frac{1}{2 d} \mathbb{1}_{\{|x|=1\}}$ as a nearest-neighbor model and to a model defined by (1.1) as a spread-out model. Furthermore, we refer to a model defined by $h_{L}$ with bounded domain as a finite-range model, to a model with $\sum_{x}|x|^{2+\epsilon} h_{L}(x)<\infty$ for some $\epsilon>0$ as a finite-variance model, and to a model with $h_{L}(x) \asymp|x|^{-d-\alpha}$ (i.e., $|x|^{d+\alpha} h_{L}(x)$ is bounded away from zero and infinity) for large $|x|$ as a long-range model with index $\alpha>0$. Obviously, finite-range models and long-range models with index $\alpha>2$ are finite-variance models.

\subsubsection{Self-avoiding walk (SAW)}

We denote by $\vec{w}_{n}$ an ordered sequence $\left(w_{0}, w_{1}, \ldots, w_{n}\right)$ of sites in $\mathbb{Z}^{d}$, and say that $\vec{w}_{n}$ is an SAW path if $w_{i} \neq w_{j}$ for $i \neq j$. We define the SAW two-point function by

$$
G_{p}^{\mathrm{SAW}}(x, y)=\sum_{n=0}^{\infty} p^{n} \sum_{\substack{\vec{w}_{n}=(x, \ldots, y) \\ \mathrm{SAW}}} \prod_{j=1}^{n} D\left(w_{j}-w_{j-1}\right),
$$

where, and in the rest of this article, we interpret the $n=0$ term as $\delta_{x, y}$. Since this is invariant under translation, we may suppress the notation to $G_{p}^{\mathrm{SAW}}(y-x):=G_{p}^{\mathrm{SAW}}(o, y-x)$. If the self-avoiding constraint is absent from $(1.2), G_{p}^{\mathrm{SAw}}(x)$ is simply reduced to the random-walk Green's function

$$
S_{p}(x)=\sum_{n=0}^{\infty} p^{n} \sum_{\vec{w}_{n}=(o, \ldots, x)} \prod_{j=1}^{n} D\left(w_{j}-w_{j-1}\right) \equiv \sum_{n=0}^{\infty} p^{n} D^{* n}(x),
$$

where $D^{* n}$ is the $n$-fold convolution of $D$, and $p^{0} D^{* 0}(x)=\delta_{x, o}$ by convention. If $S_{p}$ is summable, then its Fourier transform can be solved as

$$
\hat{S}_{p}(k):=\sum_{x \in \mathbb{Z}^{d}} e^{i k \cdot x} S_{p}(x)=\frac{1}{1-p \hat{D}(k)}=\frac{1}{1-p+p(1-\hat{D}(k))} .
$$

\subsubsection{Percolation}

A pair $\{x, y\}$ of sites in $\mathbb{Z}^{d}$ is called a bond, which is either occupied with probability $p D(y-x) \in[0,1]$ or vacant with probability $1-p D(y-x)$, independently of the other bonds. Since $D$ is a probability distribution, the percolation parameter $p$ is the average number of occupied bonds per site. We define $\{x \longleftrightarrow y\}$ to be the event that $x$ is connected to $y$, i.e., if either $x=y$ or there is an SAW path of occupied bonds from $x$ to $y$. We define the percolation two-point function by

$$
G_{p}^{\text {perc }}(x, y)=\mathbb{P}_{p}(x \longleftrightarrow y),
$$

where $\mathbb{P}_{p}$ is the probability distribution of the bond variables. We will denote the expectation against $\mathbb{P}_{p}$ by $\mathbb{E}_{p}$. Similarly to $G_{p}^{\mathrm{SAW}}(y-x)$, we may simply denote $G_{p}^{\text {perc }}(x, y)$ by $G_{p}^{\text {perc }}(y-x)$. 


\subsubsection{Ising model}

Let $\Lambda$ be a finite subset of $\mathbb{Z}^{d}$ containing the origin $o$, e.g., a $d$-dimensional hypercube centered at $o$. At each site $x \in \Lambda$, there is a spin variable $\varphi_{x}$ that takes values either +1 or -1 . The energy of the system is defined by

$$
H_{\Lambda}(\varphi)=-\sum_{\{x, y\} \subset \Lambda} J_{x, y} \varphi_{x} \varphi_{y}
$$

where $\boldsymbol{\varphi}:=\left\{\varphi_{x}\right\}_{x \in \Lambda}$ is a spin configuration and $\left\{J_{x, y}\right\}_{x, y \in \mathbb{Z}^{d}}$ is a collection of microscopic spin-spin couplings. We assume that $J_{x, y}=J_{o, y-x} \geq 0$ (i.e., translation-invariant and ferromagnetic) and that $\sum_{x} J_{o, x}<\infty$. Given the inverse temperature $\beta \geq 0$, we let

$$
p=\sum_{x \in \mathbb{Z}^{d}} \tanh \left(\beta J_{o, x}\right), \quad D(x)=\frac{1}{p} \tanh \left(\beta J_{o, x}\right) .
$$

We use $p$ as the parameter of the model, since it is increasing in $\beta<\infty$ given $\left\{J_{o, x}\right\}_{x \in \mathbb{Z}^{d}}$. We define the Ising two-point function $G_{p}^{\text {Ising }}(x, y)$ as the unique infinite-volume limit of the thermal average $\left\langle\varphi_{x} \varphi_{y}\right\rangle_{p ; \Lambda}$ :

$$
\left\langle\varphi_{x} \varphi_{y}\right\rangle_{p ; \Lambda}=\frac{\sum_{\varphi \in\{ \pm 1\}^{\Lambda}} \varphi_{x} \varphi_{y} e^{-\beta H_{\Lambda}(\varphi)}}{\sum_{\varphi \in\{ \pm 1\}^{\Lambda}} e^{-\beta H_{\Lambda}(\boldsymbol{\varphi})}}, \quad G_{p}^{\mathrm{Ising}}(x, y)=\lim _{\Lambda \uparrow \mathbb{Z}^{d}}\left\langle\varphi_{x} \varphi_{y}\right\rangle_{p ; \Lambda}
$$

where the uniqueness is assured by the second Griffiths inequality (e.g., [11]). We may denote $G_{p}^{\text {Ising }}(x, y)$ by $G_{p}^{\text {Ising }}(y-x)$, due to its translation-invariance (e.g., [6]).

\subsection{Phase transition}

Let $d \geq 2$. It is known that there is a model-dependent critical point $p_{\mathrm{c}} \in(0, \infty)$ such that the susceptibility

$$
\chi_{p}:=\left\|G_{p}\right\|_{1} \equiv \sum_{x \in \mathbb{Z}^{d}} G_{p}(x)
$$

is finite if $p<p_{\mathrm{c}}$, and infinite if $p>p_{\mathrm{c}}$. For percolation, in particular, $\chi_{p}$ is the expected number of sites connected from the origin $o: \chi_{p}=\mathbb{E}_{p}\left[\sum_{x} \mathbb{1}_{\{o \longleftrightarrow x\}}\right]$. We denote $p_{\mathrm{c}}$ for each model by $p_{\mathrm{c}}^{\mathrm{SAW}}, p_{\mathrm{c}}^{\text {perc }}$ or $p_{\mathrm{c}}^{\text {Ising }}$ if necessary. By (1.2)-(1.3) and $(1.5), 1 \leq p_{\mathrm{c}}^{\mathrm{SAW}} \leq p_{\mathrm{c}}^{\text {perc }}$; by (3.39) below, $p_{\mathrm{c}}^{\text {Ising }} \geq 1$. For percolation and the Ising model, $p_{\mathrm{c}}$ can also be characterized by the positivity of the percolation probability (i.e., the probability of existence of an infinite cluster of occupied bonds at the origin) [2] or the spontaneous magnetization (i.e., the infinite-volume limit of $\left\langle\varphi_{o}\right\rangle_{p ; \Lambda}^{+}$under the plus-boundary condition) [3].

For the finite-range models, in particular, the finiteness of $\chi_{p}$ is equivalent to exponential decay of correlation: for every $p<p_{\mathrm{c}}$, there are $C_{p}<\infty$ (which is 1 for percolation and the Ising model) and $m_{p}>0$ such that

$$
G_{p}(x) \leq C_{p} e^{-m_{p}\|x\|_{\infty}} \quad\left(x \in \mathbb{Z}^{d}\right) .
$$

This is a result of partial removal of the self-avoiding constraint [24] or of the Simon-Lieb inequality $[23,26]$. 


\subsection{Critical exponents and their universality}

The susceptibility $\chi_{p}$ is infinite not only for $p>p_{\mathrm{c}}$, but also in the limit $p \uparrow p_{\mathrm{c}}$ (e.g., $[1,4,24])$. In addition, $m_{p}$ in (1.10) tends to zero as $p \uparrow p_{\mathrm{c}}$. It is expected that there are model-dependent critical exponents $\gamma$ and $\eta$ such that

$$
\chi_{p} \underset{p \uparrow p_{\mathrm{c}}}{\approx}\left(p_{\mathrm{c}}-p\right)^{-\gamma}, \quad \hat{G}_{p_{\mathrm{c}}}(k):=\lim _{p \uparrow p_{\mathrm{c}}} \hat{G}_{p}(k) \underset{|k| \downarrow 0}{\approx}(1-\hat{D}(k))^{-1+\eta},
$$

in some appropriate sense of limits (e.g., convergence of the log-ratio of the left-hand side to the right-hand side). Other observables, such as the percolation probability and the spontaneous magnetization, are also expected to exhibit power-law behavior near $p=p_{\mathrm{c}}$ characterized by their own critical exponents. To prove existence of these critical exponents and identify their values is one of the most important problems in statistical physics. So far, there is no general proof of existence. The values of the critical exponents for two-dimensional models can be identified assuming that scaling limits of interfaces and critical cluster boundaries of the models are represented by the Schramm-Loewner Evolution (e.g., [29]). The assumption has been proved affirmative for some special cases, such as nearest-neighbor site percolation on the triangular lattice (e.g., [9]).

The most important prediction about the critical exponents is their universality. The critical exponents are believed to depend only on $d$ (and possibly on $\alpha$ for the long-range models with index $\alpha \leq 2$ ) and to be insensitive to the microscopic details, such as the value of the spread-out parameter $L$. For example, the value of $\gamma^{\text {Ising }}$ for the nearestneighbor Ising model is believed to be equal to that for the finite-variance Ising model for every $d \geq 2$, independently of $L<\infty$. It is natural to believe so, because the critical exponents characterize macroscopic physics, which should not be very much affected by the difference in the microscopic coupling $D$ of the finite-variance models (the long-range models with index $\alpha \leq 2$ may not be in the same universality class). Therefore, the critical exponents are considered to possess the intrinsic nature of the models.

As described below, the lace-expansion approach provides some evidence to believe in universality, in high dimensions.

\subsection{Mean-field behavior above the upper-critical dimension}

It is the interaction among constituents (such as spins) of each model that makes investigation of critical behavior difficult. For SAW, for example, if the effect of the selfavoiding constraint were negligible, then we could approximate $G_{p}^{\mathrm{SAW}}(x)$ by the randomwalk Green's function $S_{p}(x)$ and expect that $p_{\mathrm{c}}^{\mathrm{SAW}} \simeq 1, \gamma^{\mathrm{SAW}} \simeq 1$ and $\eta^{\mathrm{SAW}} \simeq 0$, due to (1.4). Here, we call the critical behavior described by the underlying random walk as the mean-field behavior.

However, the mean-field approximation is problematic, because of the following two reasons. (i) By ignoring the interaction among constituents, $p_{\mathrm{c}}$ decreases towards the mean-field value 1 , so we cannot really observe the behavior around the true $p_{\mathrm{c}}$. (ii) In lower dimensions, the critical exponents are expected to take on non-mean-field values; e.g., $\left(\gamma^{\mathrm{SAW}}, \eta^{\mathrm{SAW}}\right)=\left(\frac{43}{32}, \frac{5}{24}\right),\left(\gamma^{\text {perc }}, \eta^{\text {perc }}\right)=\left(\frac{43}{18}, \frac{5}{24}\right)$ and $\left(\gamma^{\text {Ising }}, \eta^{\text {Ising }}\right)=\left(\frac{7}{4}, \frac{1}{4}\right)$ in twodimensions (see $[11,24,28]$ and references therein). Let $d_{c}$ denote the model-dependent threshold dimension above which the critical exponents take on their mean-field values. If $d_{\mathrm{c}}=\infty$, it is hopeless to use the mean-field approximation to investigate the critical behavior. 
There is a sufficient condition for the mean-field behavior. Let

$$
\begin{aligned}
& B_{p}=\left(G_{p} * G_{p}\right)(o) \equiv \sum_{x \in \mathbb{Z}^{d}} G_{p}(x)^{2}, \\
& T_{p}=\left(G_{p} * G_{p} * G_{p}\right)(o) \equiv \sum_{x, y \in \mathbb{Z}^{d}} G_{p}(x) G_{p}(x, y) G_{p}(y),
\end{aligned}
$$

and say that the bubble (resp., triangle) condition holds if $B_{p_{\mathrm{c}}}<\infty$ (resp., $T_{p_{\mathrm{c}}}<\infty$ ). It is known that $\gamma$ and various other critical exponents take on their mean-field values if the bubble condition holds for SAW and the Ising model and if the triangle condition holds for percolation (see $[11,13,24]$ and references therein). This is because, for example (see $[24])$,

$$
\frac{\left(\chi_{p}^{\mathrm{SAW}}\right)^{2}}{B_{p}^{\mathrm{SAW}}} \leq \frac{\mathrm{d}\left(p \chi_{p}^{\mathrm{SAW}}\right)}{\mathrm{d} p} \leq\left(\chi_{p}^{\mathrm{SAW}}\right)^{2} \quad\left(0<p<p_{\mathrm{c}}^{\mathrm{SAW}}\right) .
$$

We obtain $\gamma^{\mathrm{SAW}}=1$ by integrating this differential inequality under the bubble condition (and using the monotonicity of $B_{p}^{\mathrm{SAW}}$ in $p$ ).

On the other hand, if there is a parameter change $\mu_{p} \in[0,1]$ with $\mu_{p_{\mathrm{c}}}=1$ such that $G_{p} \asymp S_{\mu_{p}}$ uniformly in $p<p_{\mathrm{c}}$, then $B_{p_{\mathrm{c}}}<\infty$ if $d>4$ and $T_{p_{\mathrm{c}}}<\infty$ if $d>6$ for the finite-variance models, and $B_{p_{\mathrm{c}}}<\infty$ if $d>2 \alpha$ and $T_{p_{\mathrm{c}}}<\infty$ if $d>3 \alpha$ for the long-range models with index $\alpha \leq 2$. Therefore, we are led to expect that the mean-field prediction is correct in high dimensions.

As explained in the next section, the lace expansion proves this consistency for the nearest-neighbor models in sufficiently high dimensions and for the spread-out models with $L \gg 1$ above the model-dependent upper-critical dimension.

Before closing this section, we stress that the exponential decay (1.10) does not prove the bubble/triangle condition. For example, since $m_{p} \rightarrow 0$ as $p \uparrow p_{\mathrm{c}}$ as mentioned earlier,

$$
B_{p_{\mathrm{c}}}=\lim _{p \uparrow p_{\mathrm{c}}} \sum_{x \in \mathbb{Z}^{d}} G_{p}(x)^{2} \leq 1+\lim _{p \uparrow p_{\mathrm{c}}} \sum_{r=1}^{\infty} O\left(r^{d-1}\right) e^{-2 m_{p} r}=1+\lim _{p \uparrow p_{\mathrm{c}}} O\left(m_{p}^{-d}\right)=\infty .
$$

\section{Results of the lace expansion}

In this section, we summarize some of the most intriguing results of the lace expansion.

The lace-expansion approach was initiated by Brydges and Spencer [8] for investigation of nearest-neighbor weakly SAW for $d>4$. Since then, it has been applied successfully to various finite-variance statistical-mechanical models, such as strictly SAW for $d>4$, lattice trees and lattice animals for $d>8$, percolation for $d>6$, oriented percolation and the contact process for $d>4$. See [27] for the references up to the year 2005. It has been growing to cover other models, such as finite-range self-interacting random-walk models $[19,22]$ and the finite-range Ising model for $d>4$ [25]. Analysis of the lace expansion has also been improved to deal with the long-range models with index $\alpha>0[10,18]$.

In general, the lace expansion for $G_{p}(x)$ is the following recursion equation (assuming its convergence):

$$
G_{p}(x)=\Delta_{p}(x)+\left(p D * G_{p}\right)(x)+\left(\Pi_{p} * G_{p}\right)(x)
$$


where $\left\{\Delta_{p}(x)\right\}_{x \in \mathbb{Z}^{d}}$ and $\left\{\Pi_{p}(x)\right\}_{x \in \mathbb{Z}^{d}}$ are the model-dependent expansion coefficients. If $\Delta_{p}(x)=\delta_{x, o}$ and $\Pi_{p}(x)=0$, then (2.1) is reduced to the recursion equation for the random-walk Green's function $S_{p}(x)$ :

$$
S_{p}(x)=\delta_{o, x}+\left(p D * S_{p}\right)(x) .
$$

It is therefore natural to expect that, assuming some nice properties of $\Delta_{p}$ and $\Pi_{p}$, we may find a suitable parameter change $\mu_{p} \in[0,1]$ such that $G_{p} \asymp S_{\mu_{p}}$ for $p \leq p_{\mathrm{c}}$.

\subsection{Main results}

Before showing the results below, we first introduce some notation. For the finite-variance models, we let $a_{d}=\frac{d}{2} \pi^{-d / 2} \Gamma\left(\frac{d}{2}-1\right.$ ) and $\sigma^{2}=\sum_{x}|x|^{2} D(x)$, hence $S_{1}(x) \sim \frac{a_{d}}{\sigma^{2}}|x|^{2-d}$ (see, e.g., $[14,15])$, and let

$$
d_{\mathrm{c}}= \begin{cases}4 & (\mathrm{SAW} \text { and the Ising model }) \\ 6 & \text { (percolation })\end{cases}
$$

For the long-range models with index $\alpha \leq 2$, we let

$$
d_{\mathrm{c}}= \begin{cases}2 \alpha & (\text { SAW and the Ising model }) \\ 3 \alpha & \text { (percolation })\end{cases}
$$

Let

$$
\lambda= \begin{cases}d^{-1} & (\text { nearest-neighbor models }) \\ L^{-d} & \text { (spread-out models })\end{cases}
$$

Theorem 2.1 ([16, 17, 18]). Let $d>d_{\mathrm{c}}$. For the nearest-neighbor models with $d \gg 1$ and the spread-out models with $L \gg 1$,

$$
\hat{G}_{p}(k)=\frac{1+O(\lambda)}{\chi_{p}^{-1}+p(1-\hat{D}(k))}
$$

holds uniformly in $p<p_{c}$ and $k \in[-\pi, \pi]^{d}$. Consequently, $\gamma=1$ and $\eta=0$, and various other critical exponents take on their respective mean-field values.

Theorem 2.2 ([14, 15, 25]). Let $d>d_{\mathrm{c}}$. For the nearest-neighbor models with $d \gg 1$ and the finite-range spread-out models with $L \gg 1$,

$$
G_{p_{c}}(x) \sim \frac{K a_{d}}{\sigma^{2}}|x|^{2-d} \quad \text { as }|x| \rightarrow \infty,
$$

where $K \in(0, \infty)$ is a model-dependent constant.

Theorem $2.3([20])$. Let $d>d_{\mathrm{c}}$. For the finite-range models, as $L \rightarrow \infty$,

$$
p_{c}=1+ \begin{cases}\sum_{n=2}^{\infty} D^{* n}(o)+O\left(\lambda^{2}\right) & (\text { SAW }), \\ D^{* 2}(o)+\sum_{n=3}^{\infty} \frac{n+1}{2} D^{* n}(o)+O\left(\lambda^{2}\right) & (\text { percolation }),\end{cases}
$$

where $\sum_{n=2}^{\infty} D^{* n}(o)$ and $D^{* 2}(o)+\sum_{n=3}^{\infty} \frac{n+1}{2} D^{* n}(o)$ are both $O(\lambda)$. 


\section{Remark:}

1. Theorems 2.1-2.2 hold for nearest-neighbor SAW as soon as $d \geq 5[14,17]$.

2. Theorem 2.2 may be extended to the finite-variance models, following the argument in $[14]$.

3. Similar asymptotic results to (2.8) have been obtained for spread-out oriented percolation, where $p_{\mathrm{c}}-1 \sim \frac{1}{2} \sum_{n=2}^{\infty} D^{* 2 n}(o)[10,20]$, and for the finite-range contact process, where $p_{\mathrm{c}}-1 \sim \sum_{n=2}^{\infty} D^{* n}(o)$ [20]. It is expected that $p_{\mathrm{c}}^{\text {Ising }}$ obeys the same asymptotics as $p_{\mathrm{c}}^{\mathrm{SAW}}$ in $(2.8)$, with a different error term.

\section{$2.2 \quad$ Idea of the proof}

Since the lace expansion (2.1) is a convolution equation, a natural approach to investigate it is to take its Fourier transform and solve the resulting equation for $\hat{G}_{p}(k)$. For SAW, in particular, $\hat{G}_{p}^{\mathrm{SAW}}(k)$ is considered as the Fourier-Laplace transform of the $n$-step SAW two-point function $Z_{n}^{\mathrm{SAW}}(x)$ (= the sum in (1.2) over SAWs $\vec{w}_{n}=(o, \ldots, x)$ ), where $p$ is the fugacity. Therefore, one way to investigate $\hat{Z}_{n}(k)$ for fixed $n$ is to use the Tauberian theorem to find the coefficient of $p^{n}$ in $\hat{G}_{p}^{\mathrm{SAw}}(k)$. Another way is to solve a recursion equation for $\hat{Z}_{n}^{\mathrm{SAW}}(k)$, which is a version of $(2.1)$ for fixed $n$, by induction on $n$. To investigate asymptotic behavior of $G_{p}(x)$ as $|x| \rightarrow \infty$, we may reorganize (2.1) to approximate $G_{p}(x)$ by $\kappa S_{\mu}(x)$ for some $\kappa \in(0, \infty)$ and $\mu \in[0,1]$. See [27] and references therein for more details of those approaches. Here, we focus on the proof of Theorem 2.1.

First, we heuristically explain the required properties of $\Delta_{p}$ and $\Pi_{p}$. Suppose that $\Delta_{p}$ and $\Pi_{p}$ are absolutely summable, hence $\hat{\Delta}_{p}(k)$ and $\hat{\Pi}_{p}(k)$ exist (we assume more below). Then, by solving the Fourier transform of $(2.1)$ for $\hat{G}_{p}(k)$,

$$
\begin{aligned}
\hat{G}_{p}(k) & =\hat{\Delta}_{p}(k)+p \hat{D}(k) \hat{G}_{p}(k)+\hat{\Pi}_{p}(k) \hat{G}_{p}(k) \\
& =\frac{\hat{\Delta}_{p}(k)}{1-p \hat{D}(k)-\hat{\Pi}_{p}(k)}=\frac{\hat{\Delta}_{p}(k)}{\hat{\Delta}_{p}(o) \chi_{p}^{-1}+p(1-\hat{D}(k))+\hat{\Pi}_{p}(o)-\hat{\Pi}_{p}(k)},
\end{aligned}
$$

where we have used $\hat{\Delta}_{p}(o) \chi_{p}^{-1} \equiv \hat{\Delta}_{p}(o) \hat{G}_{p}(o)^{-1}=1-p-\hat{\Pi}_{p}(o)$. We obtain (2.6) if there are $p$-independent constants $C, C^{\prime}<\infty$ such that

$$
\left.\begin{array}{c}
\left|\hat{\Delta}_{p}(k)-1\right| \\
\left|\hat{\Pi}_{p}(k)\right|
\end{array}\right\} \leq C \lambda, \quad\left|\hat{\Pi}_{p}(o)-\hat{\Pi}_{p}(k)\right| \leq C^{\prime} \lambda p(\underbrace{1-\mu_{p} \hat{D}(k)}_{\hat{S}_{\mu_{p}}(k)^{-1}})
$$

where

$$
\mu_{p}=1-\chi_{p}^{-1} \in[0,1]
$$

is a parameter change to compare $\hat{G}_{p}(k)$ with $\hat{S}_{\mu_{p}}(k)$ (cf., $f_{p}^{(1)}$ in (2.13) below); due to this definition, $\hat{G}_{p}(o)=\hat{S}_{\mu_{p}}(o)$ for any $p<p_{\mathrm{c}}$. The first inequality in (2.10) means, in a weak sense, that $\Delta_{p}(x) \simeq \delta_{x, o}$ and $\Pi_{p}(x) \simeq 0$ (cf., the discussion around (2.1)-(2.2)).

The above heuristic argument shows that (2.10) implies (2.6). To complete the proof of Theorem 2.1, it suffices to prove the converse statement: (2.6) implies (2.10). In fact, 
the actual proof is based on bootstrapping argument. To explain its latest version in $[7,18,27]$, we define

$$
f_{p}=p \vee f_{p}^{(1)} \vee f_{p}^{(2)} \geq 0
$$

where

$$
\begin{aligned}
f_{p}^{(1)} & =\sup _{k \in[-\pi, \pi]^{d}} \frac{\hat{G}_{p}(k)}{\hat{S}_{\mu_{p}}(k)}, \\
f_{p}^{(2)} & =\sup _{k, l \in[-\pi, \pi]^{d}} \frac{\hat{S}_{\mu_{p}}(k)\left|\hat{G}_{p}(l)-\frac{1}{2}\left(\hat{G}_{p}(l+k)+\hat{G}_{p}(l-k)\right)\right|}{100 \sum_{\left(j, j^{\prime}\right)=(0, \pm 1),(1,-1)} \hat{S}_{\mu_{p}}(l+j k) \hat{S}_{\mu_{p}}\left(l+j^{\prime} k\right)} .
\end{aligned}
$$

The function $f_{p}^{(2)}$ is to compare the Fourier transform of $(1-\cos (k \cdot x)) G_{p}(x)$, which is

$$
\sum_{x \in \mathbb{Z}^{d}} e^{i l \cdot x}(1-\cos (k \cdot x)) G_{p}(x)=\hat{G}_{p}(l)-\frac{1}{2}\left(\hat{G}_{p}(l+k)+\hat{G}_{p}(l-k)\right)
$$

with a constant $(=100)$ multiple of

$$
\hat{S}_{\mu_{p}}(k)^{-1} \sum_{\left(j, j^{\prime}\right)=(0, \pm 1),(1,-1)} \hat{S}_{\mu_{p}}(l+j k) \hat{S}_{\mu_{p}}\left(l+j^{\prime} k\right) .
$$

It has been proved $[7,18,27]$ (and we will demonstrate a part of (i) in Section 3) that

(i) $f_{p} \leq 3$ implies (2.10) for every $p<p_{\text {c }}$ if $d>d_{\text {c }}$ and $\lambda \ll 1$;

(ii) $f_{p} \leq 3$ and (2.10) imply the stronger bound $f_{p} \leq 2$ for every $p<p_{\mathrm{c}}$ if $d>d_{\mathrm{c}}$ and $\lambda \ll 1$

(iii) $f_{p}$ is continuous in $p<p_{\mathrm{c}}$, with $f_{0}=1$.

Because of (i)-(ii), $f_{p} \notin(2,3]$ for every $p<p_{\mathrm{c}}$. With the help of (iii), $f_{p} \leq 2$ for all $p<p_{\mathrm{c}}$, hence (2.10) indeed holds uniformly in $p<p_{\mathrm{c}}$. This proves Theorem 2.1.

\section{Lace expansion and bounding diagrams}

In this section, we explain (the first few stages of) the derivation of the lace expansion (2.1) for each model. We note here that there are many identities of the form (2.1) depending on $\Delta_{p}$ and $\Pi_{p}$. Among those identities, the lace expansion is the one with optimal $\Delta_{p}$ and $\Pi_{p}$, which means that $\Delta_{p}$ and $\Pi_{p}$ are absolutely summable for $p \leq p_{\mathrm{c}}$ (cf., the mean-field approximation, which is valid only for $p<1$ ) and $d>d_{\mathrm{c}}$. We briefly explain that $\Delta_{p}$ and $\Pi_{p}$ are bounded by geometrical series of nonzero bubbles for SAW and the Ising model and of nonzero triangles for percolation, where the nonzero bubble/triangle is $O(\lambda)$ if $d>d_{\mathrm{c}}$. 


\subsection{Expansion for self-avoiding walk}

In this section, we use inclusion-exclusion to derive the expansion (2.1) for $G_{p}^{\mathrm{SAW}}(x)$, with $\Delta_{p}^{\mathrm{SAW}}(x)=\delta_{x, o}$ and

$$
\Pi_{p}^{\mathrm{SAW}}(x)=\sum_{N=1}^{\infty}(-1)^{N} \Pi_{p ; N}^{\mathrm{SAW}}(x) \equiv-\bigodot_{x=0}+\bigodot_{o}^{x}-\bigodot_{o}+\cdots
$$

where each $\Pi_{p ; N}^{\mathrm{SAW}}(x) \geq 0$ consists of $N$ vertices and $2 N-1$ lines; unlabeled vertices in $\Pi_{p ; N}^{\mathrm{SAW}}(x)$ for $N \geq 3$ are summed over $\mathbb{Z}^{d}$; unslashed lines correspond to nonzero SAW paths (or loop when $N=1$ ), while slashed lines in $\Pi_{p ; N}^{\mathrm{SAW}}(x)$ for $N \geq 3$ correspond to SAW paths which may have zero length. Some lines in each term are mutually avoiding, others are not. For example, all the lines in $\Pi_{p ; 2}^{\mathrm{SAW}}(x)$ are mutually avoiding, which is not the case for $\Pi_{p ; 3}^{\mathrm{SAW}}(x)$. Refer to, e.g., [24] for the subtlety of this mutual avoidance.

Before showing the derivation of the lace expansion, we demonstrate how $f_{p} \leq 3$ implies the inequalities in (2.10) for $\Pi_{p}^{\mathrm{SAW}}$ if $d>d_{\mathrm{c}}^{\mathrm{SAW}}$ and $\lambda \ll 1$. First, by ignoring the mutual avoidance among consisting SAW paths, $\left|\hat{\Pi}_{p}^{\mathrm{SAW}}(k)\right|$ is bounded as

$$
\left|\hat{\Pi}_{p}^{\mathrm{SAW}}(k)\right| \leq \sum_{x \in \mathbb{Z}^{d}}\left(\bigodot_{x=0}+\tilde{G}_{p}^{\mathrm{SAW}}(x)^{3}+G_{p}^{\mathrm{SAW}}(x)\left(\left(\tilde{G}_{p}^{\mathrm{SAW}}\right)^{2} *\left(\tilde{G}_{p}^{\mathrm{SAW}}\right)^{2}\right)(x)+\cdots\right)
$$

where $\tilde{G}_{p}^{\mathrm{SAW}}(x)=G_{p}^{\mathrm{SAW}}(x)-\delta_{x, o}$, which obeys the trivial bound

$$
\tilde{G}_{p}^{\mathrm{SAW}}(x) \leq\left(p D * G_{p}^{\mathrm{SAW}}\right)(x) \leq p D(x)+p^{2}\left(D^{* 2} * G_{p}^{\mathrm{SAW}}\right)(x) .
$$

If $p \vee f_{p}^{(1)} \leq 3$ and $d>\frac{1}{2} d_{\mathrm{c}}^{\mathrm{SAW}}$, then the sum of the first term in (3.2) is bounded as

$$
\begin{aligned}
& \sum_{x \in \mathbb{Z}^{d}} \bigcirc_{x=o} \leq p^{2}\left(D^{* 2} * G_{p}^{\mathrm{SAw}}\right)(o)=p^{2} \int_{[-\pi, \pi]^{d}} \frac{\mathrm{d}^{d} k}{(2 \pi)^{d}} \hat{D}(k)^{2} \hat{G}_{p}^{\mathrm{SAw}}(k) \\
& \quad \leq 3^{3} \int_{[-\pi, \pi]^{d}} \frac{\mathrm{d}^{d} k}{(2 \pi)^{d}} \hat{D}(k)^{2} \hat{S}_{\mu_{p}}(k) \leq 3^{3} \int_{[-\pi, \pi]^{d}} \frac{\mathrm{d}^{d} k}{(2 \pi)^{d}} \frac{\hat{D}(k)^{2}}{1-\hat{D}(k)}=O(\lambda),
\end{aligned}
$$

where we have used the random-walk estimates (see, e.g., [27] for the nearest-neighbor model and $[10,20,21]$ for the spread-out model). For the sum of the second term in (3.2), we use

$$
\sum_{x \in \mathbb{Z}^{d}} \tilde{G}_{p}^{\mathrm{SAW}}(x)^{3} \leq\left\|\tilde{G}_{p}^{\mathrm{SAW}}\right\|_{\infty}\left(\tilde{G}_{p}^{\mathrm{SAW}}\right)^{* 2}(o)
$$

where $\left\|\tilde{G}_{p}^{\mathrm{SAw}}\right\|_{\infty}=O(\lambda)$ if $d>\frac{1}{2} d_{\mathrm{c}}^{\mathrm{SAw}}$, due to $(3.3),\|D\|_{\infty}=O(\lambda)$ and a similar analysis to (3.4). Moreover, if $d>d_{\mathrm{c}}^{\mathrm{SAW}}$,

$$
\begin{aligned}
& \left(\tilde{G}_{p}^{\mathrm{SAW}}\right)^{* 2}(o) \leq p^{2}\left(D * G_{p}^{\mathrm{SAW}}\right)^{* 2}(o)=p^{2} \int_{[-\pi, \pi]^{d}} \frac{\mathrm{d}^{d} k}{(2 \pi)^{d}} \hat{D}(k)^{2} \hat{G}_{p}^{\mathrm{SAW}}(k)^{2} \\
& \leq 3^{4} \int_{[-\pi, \pi]^{d}} \frac{\mathrm{d}^{d} k}{(2 \pi)^{d}} \hat{D}(k)^{2} \hat{S}_{\mu_{p}}(k)^{2} \leq 3^{4} \int_{[-\pi, \pi]^{d}} \frac{\mathrm{d}^{d} k}{(2 \pi)^{d}} \frac{\hat{D}(k)^{2}}{(1-\hat{D}(k))^{2}}=O(\lambda) .
\end{aligned}
$$


Therefore, $\left\|\Pi_{p ; 2}^{\mathrm{SAW}}\right\|_{1} \leq O(\lambda)^{2}$. By similar manipulation of supremums and sums, we can show $\left\|\Pi_{p ; N}^{\mathrm{SAW}}\right\|_{1} \leq O(\lambda)^{N}$ if $d>d_{\mathrm{c}}^{\mathrm{SAW}}$ (see, e.g., [24]), hence the series (3.2) converges and is $O(\lambda)$ if $\lambda \ll 1$. This proves the first inequality in (2.10).

For the second inequality in (2.10), we use $f_{p}^{(2)}$ as well. For example,

$$
\begin{aligned}
\hat{\Pi}_{p ; 2}^{\mathrm{SAW}}(o)-\hat{\Pi}_{p ; 2}^{\mathrm{SAW}}(k) & =\sum_{x \in \mathbb{Z}^{d}}(1-\cos (k \cdot x)) \Pi_{p ; 2}^{\mathrm{SAW}}(x) \leq \sum_{x \in \mathbb{Z}^{d}}(1-\cos (k \cdot x)) \tilde{G}_{p}^{\mathrm{SAW}}(x)^{3} \\
& \leq\left(\sup _{x \in \mathbb{Z}^{d}}(1-\cos (k \cdot x)) G_{p}^{\mathrm{SAW}}(x)\right) \underbrace{\left(\tilde{G}_{p}^{\mathrm{SAW}}\right)^{* 2}(o)}_{p O(\lambda)}
\end{aligned}
$$

where, by $(2.15)$ and $f_{p}^{(2)} \leq 3$,

$$
\begin{aligned}
& (1-\cos (k \cdot x)) G_{p}^{\mathrm{SAW}}(x) \leq \int_{[-\pi, \pi]^{d}} \frac{\mathrm{d}^{d} l}{(2 \pi)^{d}}\left|\hat{G}_{p}^{\mathrm{SAW}}(l)-\frac{1}{2}\left(\hat{G}_{p}^{\mathrm{SAW}}(l+k)+\hat{G}_{p}^{\mathrm{SAw}}(l-k)\right)\right| \\
& \quad \leq \sum_{\left(j, j^{\prime}\right)=(0, \pm 1),(1,-1)} \int_{[-\pi, \pi]^{d}} \frac{\mathrm{d}^{d} l}{(2 \pi)^{d}} \frac{300 \hat{S}_{\mu_{p}}(k)^{-1}}{(1-\hat{D}(l+j k))\left(1-\hat{D}\left(l+j^{\prime} k\right)\right)}
\end{aligned}
$$

which is $O(1) \hat{S}_{\mu_{p}}(k)^{-1}$ if $d>d_{\mathrm{c}}^{\mathrm{SAw}}$, hence $\hat{\Pi}_{p ; 2}^{\mathrm{SAW}}(o)-\hat{\Pi}_{p ; 2}^{\mathrm{SAW}}(k) \leq O(\lambda) p \hat{S}_{\mu_{p}}(k)^{-1}$. Similarly, we can show $\hat{\Pi}_{p ; N}^{\mathrm{SAW}}(o)-\hat{\Pi}_{p ; N}^{\mathrm{SAW}}(k) \leq O(\lambda)^{N} p \hat{S}_{\mu_{p}}(k)^{-1}$ if $d>d_{\mathrm{c}}^{\mathrm{SAW}}[18,27]$, which yields the second inequality in $(2.10)$ if $\lambda \ll 1$.

Derivation of the lace expansion for $G_{p}^{\mathrm{SAw}}(x)$. We only explain the first two stages of the derivation of the expansion (2.1). To complete the expansion, we refer to, e.g., [24].

The first stage of the expansion is to isolate the zero-step walk and identify the position of $w_{1}$ for the nonzero SAW paths $\vec{w}_{n}=\left(o, w_{1}, \ldots, x\right)$. Denoting these nonzero SAW paths by $\vec{w}_{n}=\left(o, \vec{w}_{n-1}^{\prime}\right)$, we obtain

$$
\begin{aligned}
G_{p}^{\mathrm{SAW}}(x) & =\delta_{x, o}+\sum_{n=1}^{\infty} p^{n} \sum_{y \in \mathbb{Z}^{d}} \sum_{\vec{w}_{n}=(o, y, \ldots, x)} \prod_{j=1}^{n} D\left(w_{j}-w_{j-1}\right) \\
& =\delta_{x, o}+\sum_{y \in \mathbb{Z}^{d}} p D(y) \sum_{n=1}^{\infty} p^{n-1} \sum_{\substack{\vec{w}_{n-1}^{\prime}=(y, \ldots, x) \\
\mathrm{SAW}}} \prod_{j=1}^{n-1} D\left(w_{j}^{\prime}-w_{j-1}^{\prime}\right) \mathbb{1}_{\left\{\vec{w}_{n-1}^{\prime} \not \supset o\right\}} .
\end{aligned}
$$

Since $\vec{w}_{n-1}^{\prime}$ is an SAW path, the last indicator is 1 if and only if $\vec{w}_{n}=\left(o, \vec{w}_{n-1}^{\prime}\right)$ is an SAW path. If this indicator is absent, then the sum over $n$ is simply equal to $G_{p}^{\mathrm{SAW}}(y, x)$; the correction is the contribution from $\mathbb{1}_{\left\{\vec{w}_{n-1}^{\prime} \ni o\right\}} \equiv 1-\mathbb{1}_{\left\{\vec{w}_{n-1}^{\prime} \not \supset o\right\}}$, due to inclusion-exclusion. Therefore,

$$
G_{p}^{\mathrm{SAW}}(x)=\delta_{x, o}+\left(p D * G_{p}^{\mathrm{SAW}}\right)(x)-R_{p ; 1}^{\mathrm{SAW}}(x)
$$

with

$$
R_{p ; 1}^{\mathrm{SAW}}(x)=\sum_{y \in \mathbb{Z}^{d}} p D(y) \sum_{n=0}^{\infty} p^{n} \sum_{\substack{\vec{w}_{n}=(y, \ldots, o, \ldots, x) \\ \mathrm{SAW}}} \prod_{j=1}^{n} D\left(w_{j}-w_{j-1}\right)
$$


where we have replaced $n-1$ in (3.9) by $n^{\prime} \in \mathbb{Z}_{+}:=\{0,1, \ldots\}$ and then removed all the primes from the expression. The derivation of the full expansion is completed if we can show $R_{p ; 1}^{\mathrm{SAW}}(x)=-\left(\Pi_{p}^{\mathrm{SAW}} * G_{p}^{\mathrm{SAW}}\right)(x)$.

The second stage of the expansion is to split an SAW path $\vec{w}_{n}=(y, \ldots, o, \ldots, x)$ in (3.11) into two subwalks according to the "time" $t$ at which $\vec{w}_{n}$ is at $o$. Let $\vec{w}_{n}=\left(\vec{w}_{t}, \vec{w}_{n-t}^{\prime}\right)$, where $\vec{w}_{t}=(y, \ldots, o)$ and $\vec{w}_{n-t}^{\prime}=(o, \ldots, x)$. Then, we obtain

$$
\begin{aligned}
R_{p ; 1}^{\mathrm{SAW}}(x)=\sum_{y \in \mathbb{Z}^{d}} p D(y) \sum_{t=0}^{\infty} p^{t} \sum_{\substack{\vec{w}_{t}=(y, \ldots, o) \\
\mathrm{SAW}}} \prod_{i=1}^{t} D\left(w_{i}-w_{i-1}\right) \\
\times \sum_{n=t}^{\infty} p^{n-t} \sum_{\substack{\vec{w}_{n-t}^{\prime}=(o, \ldots, x) \\
\mathrm{SAW}}} \prod_{j=1}^{n-t} D\left(w_{j}^{\prime}-w_{j-1}^{\prime}\right) \mathbb{1}_{\left\{\vec{w}_{t} \cap \vec{w}_{n-t}^{\prime}=\{o\}\right\}} .
\end{aligned}
$$

Since $\vec{w}_{t}$ and $\vec{w}_{n-t}^{\prime}$ are SAW paths, the last indicator is 1 if and only if $\vec{w}_{n}=\left(\vec{w}_{t}, \vec{w}_{n-t}^{\prime}\right)$ is an SAW path. If we ignore this indicator, the first and second lines become independent, yielding $\left(\Pi_{p ; 1}^{\mathrm{SAW}} * G_{p}^{\mathrm{SAW}}\right)(x)$, where $\Pi_{p ; 1}^{\mathrm{SAW}}(x) \equiv \bigcap_{x=0}$ is the self-avoiding loop at the origin. The correction is the contribution from $\mathbb{1}_{\left\{\vec{w}_{t} \cap \vec{w}_{n-t}^{\prime} \supsetneq\{o\}\right\}} \equiv 1-\mathbb{1}_{\left\{\vec{w}_{t} \cap \vec{w}_{n-t}^{\prime}=\{o\}\right\}}$. Therefore,

$$
R_{p ; 1}^{\mathrm{SAW}}(x)=\left(\Pi_{p ; 1}^{\mathrm{SAW}} * G_{p}^{\mathrm{SAW}}\right)(x)-R_{p ; 2}^{\mathrm{SAW}}(x),
$$

where, by replacing $n-t$ in (3.12) by $s \in \mathbb{Z}_{+}$,

$$
\begin{aligned}
R_{p ; 2}^{\mathrm{SAW}}(x)=\sum_{y \in \mathbb{Z}^{d}} p D(y) & \sum_{t=0}^{\infty} p^{t} \sum_{\substack{\vec{w}_{t}=(y, \ldots, o) \\
\mathrm{SAW}}} \prod_{i=1}^{t} D\left(w_{i}-w_{i-1}\right) \\
& \times \sum_{s=0}^{\infty} p^{s} \sum_{\substack{\vec{w}_{s}^{\prime}=(o, \ldots, x) \\
\mathrm{SAW}}}^{s} \prod_{j=1}^{s} D\left(w_{j}^{\prime}-w_{j-1}^{\prime}\right) \mathbb{1}_{\left\{\vec{w}_{t} \cap \vec{w}_{s}^{\prime} \supsetneq\{o\}\right\} .}
\end{aligned}
$$

This completes the second stage of the expansion.

To obtain the higher-order expansion coefficient $\Pi_{p ; N}^{\mathrm{SAW}}(x)$, we look at the last SAW path in the higher-order remainder $R_{p ; N}^{\mathrm{SAW}}(x)$, e.g., $\vec{w}_{s}^{\prime}$ in $R_{p ; 2}^{\mathrm{SAW}}(x)$, and see when for the first time the condition in the indicator, e.g., $\vec{w}_{t} \cap \vec{w}_{s}^{\prime} \supsetneq\{o\}$ in (3.14), is satisfied. Let $u \in\{0,1 \ldots, s\}$ be that "first time" and split the path $\vec{w}_{s}^{\prime}$ into $\vec{w}_{s}^{\prime}=\left(\vec{w}_{u}^{\prime}, \vec{w}_{s-u}^{\prime \prime}\right)$. If we forget that $\vec{w}_{u}^{\prime}$ and $\vec{w}_{s-u}^{\prime \prime}$ are mutually avoiding, we obtain $\left(\Pi_{p ; N}^{\mathrm{SAW}} * G_{p}^{\mathrm{SAW}}\right)(x)$; the correction becomes $-R_{p ; N+1}^{\mathrm{SAW}}(x)$. See, e.g., [24] for more details of this inclusion-exclusion argument.

\subsection{Expansion for percolation}

Here and in Section 3.3, we explain the derivation of the following expansion for percolation and the Ising model $^{1}$ :

$$
G_{p}(x)=\Delta_{p}(x)+\left(\Delta_{p} * p D * G_{p}\right)(x) \equiv \Delta_{p}(x)+\left(p D * G_{p}\right)(x)+(\underbrace{\left(\Delta_{p}-\delta\right) * p D}_{\Pi_{p}} * G_{p})(x),
$$

\footnotetext{
${ }^{1}$ To keep this article as simple and intuitive as possible, we pretend here and in Section 3.3 that the expansion (3.15) is complete without a remainder, with the series representation (3.16) for $\Delta_{p}(x)$. See $[16,25]$ for the lace expansion up to any $\ell^{\text {th }}$ order, with $\Delta_{p}(x)=\sum_{N=0}^{\ell}(-1)^{N} \Delta_{p ; N}(x)$ and a remainder.
} 
where $\Delta_{p}(x)$ is the alternating series of the model-dependent $N^{\text {th }}$-order expansion coefficients $\Delta_{p ; N}(x) \geq 0$ :

$$
\Delta_{p}(x)=\sum_{N=0}^{\infty}(-1)^{N} \Delta_{p ; N}(x) .
$$

For percolation $[16], \Delta_{p}^{\text {perc }}(x)$ may be depicted as

$$
\Delta_{p}^{\text {perc }}(x)=o \circlearrowright x-o \circlearrowright>x+\cdots,
$$

where each line corresponds to an occupied path that may have zero length (the horizontal bold line in the second term has an occupied bond at the left end, hence it is nonzero). The first term represents the probability that $o$ is doubly-connected to $x$, i.e., either $x=o$ or there are at least two bond-disjoint occupied paths from $o$ to $x \neq o$. The higher-order terms may be interpreted in a similar way. For example, in the second term, the thinner lines are mutually bond-disjoint occupied paths, and so are the bold lines. The difference in thickness is to represent nested expectations; the bold lines are on a probability space that is different from the one the thinner lines are defined on, and these lines are coupled in order to satisfy certain geometrical conditions. The higher-order terms are also defined by using nested expectations. See, e.g., [16] for more details.

Before going into the derivation of the expansion (3.15), we demonstrate how to use $f_{p} \leq 3$ to obtain (2.10) if $d>d_{\mathrm{c}}^{\text {perc }}$ and $\lambda \ll 1$. The key is the BK inequality [5], by which we can prove that the probability of occurrence of bond-disjoint connections is bounded from above by the product of the probability of occurrence of each connection. For example, by the BK inequality,

$$
\delta_{x, o} \leq o \circlearrowright x \leq G_{p}^{\mathrm{perc}}(x)^{2}=\delta_{x, o}+\tilde{G}_{p}^{\mathrm{perc}}(x)^{2},
$$

where each $\tilde{G}_{p}^{\text {perc }}(x):=G_{p}^{\text {perc }}(x)-\delta_{x, o}$ is bounded, again by the BK inequality, as

$$
\begin{aligned}
\tilde{G}_{p}^{\text {perc }}(x) & \leq \sum_{v \in \mathbb{Z}^{d}} \mathbb{P}_{p}(\{o, v\} \text { is occupied, } v \longleftrightarrow x \text { without using }\{o, v\}) \\
& \leq\left(p D * G_{p}^{\text {perc }}\right)(x) \leq p D(x)+p^{2}\left(D^{* 2} * G_{p}^{\text {perc }}\right)(x) .
\end{aligned}
$$

Therefore, by the same computation as in (3.6), we obtain $\left|\hat{\Delta}_{p ; 0}^{\text {perc }}(k)-1\right| \leq O(\lambda)$ if $d>$ $\frac{2}{3} d_{\mathrm{c}}^{\text {perc }}$. Similarly, by the BK inequality and taking supremums and sums,

$$
\begin{aligned}
\left\|\Delta_{p ; 1}^{\mathrm{perc}}\right\|_{1} \leq \sum_{u, v \in \mathbb{Z}^{d}} G_{p}^{\mathrm{perc}}(u) G_{p}^{\mathrm{perc}}(u, v) G_{p}^{\mathrm{perc}}(v) \sum_{y, z \in \mathbb{Z}^{d}}\left(p D * G_{p}^{\mathrm{perc}}\right)(v, y) G_{p}^{\mathrm{perc}}(y, z) G_{p}^{\mathrm{perc}}(z, u) \\
\quad \times \sum_{x \in \mathbb{Z}^{d}} G_{p}^{\mathrm{perc}}(y, x) G_{p}^{\mathrm{perc}}(x, z) \\
\leq\left(G_{p}^{\mathrm{perc}}\right)^{* 3}(o)\left\|p D *\left(G_{p}^{\mathrm{perc}}\right)^{* 3}\right\|_{\infty}\left\|\left(G_{p}^{\mathrm{perc}}\right)^{* 2}\right\|_{\infty}
\end{aligned}
$$

Since $f_{p}^{(1)} \leq 3$ and $d>d_{\mathrm{c}}^{\text {perc }}$, both $\left(G_{p}^{\text {perc }}\right)^{* 3}(o)$ and $\left\|\left(G_{p}^{\text {perc }}\right)^{* 2}\right\|_{\infty}$ are $O(1)$. Moreover, by (3.19) and $G_{p}^{\text {perc }}(x) \geq \delta_{x, o}$ and using $p \vee f_{p}^{(1)} \leq 3$ and the random-walk estimates mentioned 
below (3.4),

$$
\begin{aligned}
\left\|p D *\left(G_{p}^{\text {perc }}\right)^{* 3}\right\|_{\infty} & \leq p\|D\|_{\infty}+3 p^{2}\left\|D^{* 2} *\left(G_{p}^{\text {perc }}\right)^{* 3}\right\|_{\infty} \\
& \leq 3\|D\|_{\infty}+3^{6} \int_{[-\pi, \pi]^{d}} \frac{\mathrm{d}^{d} k}{(2 \pi)^{d}} \frac{\hat{D}(k)^{2}}{(1-\hat{D}(k))^{3}}=O(\lambda),
\end{aligned}
$$

if $d>d_{\mathrm{c}}^{\text {perc }}$, hence $\left\|\Delta_{p: 1}^{\text {perc }}\right\|_{1} \leq O(\lambda)$. By similar manipulations of supremums and sums, we

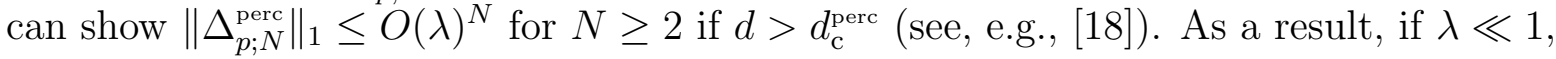

$$
\left|\hat{\Delta}_{p}^{\text {perc }}(k)-1\right| \leq\left|\hat{\Delta}_{p ; 0}^{\text {perc }}(k)-1\right|+\sum_{N=1}^{\infty}\left\|\Delta_{p ; N}^{\text {perc }}\right\|_{1}=O(\lambda),
$$

which also implies $\left|\hat{\Pi}_{p}^{\text {perc }}(k)\right| \leq O(\lambda)$. This proves the first inequality in (2.10).

For the second inequality in (2.10), we first note that

$$
\begin{aligned}
\hat{\Pi}_{p}^{\text {perc }}(o)-\hat{\Pi}_{p}^{\text {perc }}(k) & =p\left(\left(\hat{\Delta}_{p}^{\text {perc }}(o)-1\right)-\left(\hat{\Delta}_{p}^{\text {perc }}(k)-1\right) \hat{D}(k)\right) \\
& =p\left(\left(\hat{\Delta}_{p}^{\text {perc }}(o)-\hat{\Delta}_{p}^{\text {perc }}(k)\right)+\left(\hat{\Delta}_{p}^{\text {perc }}(k)-1\right)(1-\hat{D}(k))\right) .
\end{aligned}
$$

Since $1-\hat{D}(k) \leq 2 \hat{S}_{\mu_{p}}(k)^{-1}$, it thus suffices to show $\left|\hat{\Delta}_{p}^{\text {perc }}(o)-\hat{\Delta}_{p}^{\text {perc }}(k)\right| \leq O(\lambda) \hat{S}_{\mu_{p}}(k)^{-1}$. However, we only show here that $\hat{\Delta}_{p ; 0}^{\text {perc }}(o)-\hat{\Delta}_{p ; 0}^{\text {perc }}(k) \leq O(1) \hat{S}_{\mu_{p}}(k)^{-1}$ (the wanted $O(\lambda)$ term can be extracted using (3.19) [18]). By (3.18) and (2.15) and using $f_{p}^{(1)} \vee f_{p}^{(2)} \leq 3$, we obtain

$$
\begin{aligned}
& \hat{\Delta}_{p ; 0}^{\text {perc }}(o)-\hat{\Delta}_{p ; 0}^{\text {perc }}(k)=\sum_{x \in \mathbb{Z}^{d}}(1-\cos (k \cdot x)) \Delta_{p ; 0}^{\text {perc }}(x) \leq \sum_{x \in \mathbb{Z}^{d}}(1-\cos (k \cdot x)) G_{p}^{\text {perc }}(x)^{2} \\
& \quad=\int_{[-\pi, \pi]^{d}} \frac{\mathrm{d}^{d} l}{(2 \pi)^{d}} \hat{G}_{p}^{\text {perc }}(l)\left(\hat{G}_{p}^{\text {perc }}(l)-\frac{1}{2}\left(\hat{G}_{p}^{\text {perc }}(l+k)+\hat{G}_{p}^{\text {perc }}(l-k)\right)\right) \\
& \leq \sum_{\left(j, j^{\prime}\right)=(0, \pm 1),(1,-1)} \int_{[-\pi, \pi]^{d}} \frac{\mathrm{d}^{d} l}{(2 \pi)^{d}} \frac{900 \hat{S}_{\mu_{p}}(k)^{-1}}{(1-\hat{D}(l))(1-\hat{D}(l+j k))\left(1-\hat{D}\left(l+j^{\prime} k\right)\right)}
\end{aligned}
$$

which is $O(1) \hat{S}_{\mu_{p}}(k)^{-1}$ if $d>d_{\mathrm{c}}^{\text {perc }}$, as required. The higher-order term $\hat{\Delta}_{p ; N}^{\text {perc }}(o)-\hat{\Delta}_{p ; N}^{\text {perc }}(k)$ obeys a diagrammatic bound that contains $N$ nonzero triangles, which is bounded by $O(\lambda)^{N} \hat{S}_{\mu_{p}}(k)^{-1}$ for $N \geq 1$ if $d>d_{\mathrm{c}}^{\text {perc }}$ [18], yielding the second inequality in (2.10) if $\lambda \ll 1$.

Derivation of the lace expansion for $G_{p}^{\text {perc }}(x)$. Here, we only explain the first stage of the derivation of the expansion (3.15). See [16] for the completion of the expansion.

First, we introduce the following notions and notation (which will also be used for the Ising model):

Definition 3.1. $\quad$ 1. For $V \subset \mathbb{Z}^{d}$, we denote by $\mathbb{B}_{V}$ the set of bonds whose vertices are both in $V$. Given a bond configuration $\boldsymbol{n} \equiv\left\{n_{b}\right\}$ (where $n_{b}=\mathbb{1}_{\{b \text { is occupied }\}}$ ), we say that $x$ is $\boldsymbol{n}$-connected to $y$ in $V$, denoted $x \overleftrightarrow{n} y$ in $V$, if either $x=y \in V$ or there is a path from $x$ to $y$ consisting of bonds $b \in \mathbb{B}_{V}$ with $n_{b}>0$. 
2. For an event $E$ (= a set of bond configurations) and a bond $b$, the event $\{E$ without using $b\}$ is the set of bond configurations $\boldsymbol{n} \in E$ such that the new configuration obtained by changing $n_{b}$ is also in $E$. Given a bond configuration $\boldsymbol{n}$, we let

$$
\mathcal{C}_{\boldsymbol{n}}^{b}(x)=\{y: x \underset{n}{\longleftrightarrow} y \text { without using } b\} .
$$

3. We denote the head and tail of a directed bond $b$ by $\bar{b}$ and $\underline{b}$, respectively: $b=(\underline{b}, \bar{b})$. Given a bond configuration $\boldsymbol{n}$, we say that a directed bond $b$ is pivotal for $x \overleftrightarrow{n}$ from $x$, if $x \overleftrightarrow{n} \underline{b}$ without using $b$ and $\bar{b} \underset{n}{\longleftrightarrow} y$ in $\mathbb{Z}^{d} \backslash \mathcal{C}_{\boldsymbol{n}}^{b}(x)$. Let

$$
\operatorname{piv}_{\boldsymbol{n}}(x, y)=\{b: b \text { is pivotal for } x \overleftrightarrow{n} y \text { from } x\}
$$

If $x \overleftrightarrow{n} y$ and $\operatorname{piv}_{\boldsymbol{n}}(x, y)=\varnothing$, we say that $x$ is $\boldsymbol{n}$-doubly connected to $y$, denoted $x \Longleftrightarrow y$.

4. As usual, we abbreviate $\left\{\boldsymbol{n}: \mathcal{C}_{\boldsymbol{n}}^{b}(x)=V\right\}$ to $\left\{\mathcal{C}^{b}(x)=V\right\}$, and $\left\{\boldsymbol{n}: b \in \operatorname{piv}_{\boldsymbol{n}}(x, y)\right\}$ to $\{b \in \operatorname{piv}(x, y)\}$, etc.

We now begin with the derivation of the lace expansion for $G_{p}^{\text {perc }}(x) \equiv \mathbb{P}_{p}(o \longleftrightarrow x)$. First we note that, for each bond configuration $\boldsymbol{n}$, there are two possibilities for $O \overleftrightarrow{n} x$ : either $\operatorname{piv}_{\boldsymbol{n}}(o, x)=\varnothing$ or $\operatorname{piv}_{\boldsymbol{n}}(o, x) \neq \varnothing$. If $\operatorname{piv}_{\boldsymbol{n}}(o, x) \neq \varnothing$, we take its first element $b$ (so that $o \Longleftrightarrow \underline{b}$ ). Then, we have

$$
\{o \longleftrightarrow x\}=\underbrace{\{o \longleftrightarrow x, \operatorname{piv}(o, x)=\varnothing\}}_{\{o \Longleftrightarrow x\}} \dot{\cup} \bigcup_{b}\{o \longleftrightarrow x, b \in \operatorname{piv}(o, x), o \Longleftrightarrow \underline{b}\},
$$

where, by definition, the event subject to the big union over $b$ can be written as

$$
\{b \text { is occupied }\} \cap\{o \Longleftrightarrow \underline{b} \text { without using } b\} \cap\left\{\bar{b} \longleftrightarrow x \text { in } \mathbb{Z}^{d} \backslash \mathcal{C}^{b}(o)\right\} .
$$

Let $p_{b}=p D(\bar{b}-\underline{b}) \equiv \mathbb{P}_{p}(b$ is occupied $)$. Since $\{b$ is occupied $\}$ is independent of the other two events in (3.28), we obtain

$$
G_{p}^{\text {perc }}(x)=\Delta_{p ; 0}^{\text {perc }}(x)+\sum_{b} p_{b} \mathbb{E}_{p}\left[\mathbb{1}_{\{o \Longleftrightarrow \underline{b}\}} \mathbb{1}_{\left\{\bar{b} \longleftrightarrow x \text { in } \mathbb{Z}^{d} \backslash \mathcal{C}^{b}(o)\right\}}\right]
$$

where the "without using $b$ " condition has been ignored, as $\mathbb{1}_{\left\{\bar{b} \longleftrightarrow x \text { in } \mathbb{Z}^{d} \backslash \mathcal{C}^{b}(o)\right\}}$ is always zero on the event $\left\{\bar{b} \in \mathcal{C}^{b}(o)\right\} \supset\{o \Longleftrightarrow x\} \backslash\{o \Longleftrightarrow x$ without using $b\}$.

Next we investigate the expectation in (3.29). For notational convenience, we will drop the subscript $p$ from $\mathbb{P}_{p}$ and $\mathbb{E}_{p}$. First, by conditioning on the cluster $\mathcal{C}^{b}(o)$, we can formally write

$$
\begin{aligned}
\mathbb{E}\left[\mathbb{1}_{\{o \Longleftrightarrow \underline{b}\}} \mathbb{1}_{\left\{\bar{b} \longleftrightarrow x \text { in } \mathbb{Z}^{d} \backslash \mathcal{C}^{b}(o)\right\}}\right] & =\sum_{V \subset \mathbb{Z}^{d}} \mathbb{E}\left[\mathbb{1}_{\left\{\mathcal{C}^{b}(o)=V, o \Longleftrightarrow \underline{b}\right\}} \mathbb{1}_{\left\{\bar{b} \longleftrightarrow x \text { in } \mathbb{Z}^{d} \backslash V\right\}}\right] \\
& \left.=\sum_{V \subset \mathbb{Z}^{d}(\boldsymbol{l}, \boldsymbol{m})} \sum \mathbb{P}(\boldsymbol{l}, \boldsymbol{m}) \mathbb{1}_{\left\{\mathcal{C}_{l}^{b}(o)=V, o \Longleftrightarrow\right.} \underline{b}\right\} \mathbb{1}_{\{\bar{b} \overleftrightarrow{\boldsymbol{m}} x\}},
\end{aligned}
$$




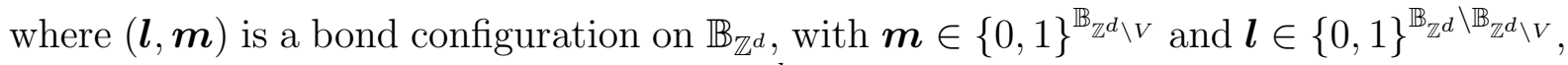
and we have ignored the unnecessary "in $\mathbb{Z}^{d} \backslash V$ " condition for such an $\boldsymbol{m}$. Let

$$
\mathbb{P}_{\mathbb{Z}^{d} \backslash V}(\boldsymbol{m})=\prod_{b \in \mathbb{B}_{\mathbb{Z}^{d} \backslash V}}\left(p_{b} \delta_{m_{b}, 1}+\left(1-p_{b}\right) \delta_{m_{b}, 0}\right)
$$

and let $\tilde{\mathbb{P}}_{V}$ be such that $\mathbb{P}(\boldsymbol{l}, \boldsymbol{m})=\tilde{\mathbb{P}}_{V}(\boldsymbol{l}) \mathbb{P}_{\mathbb{Z}^{d} \backslash V}(\boldsymbol{m})$. Then, by multiplying and dividing (3.30) by $\sum_{\boldsymbol{m}^{\prime}} \mathbb{P}_{\mathbb{Z}^{d} \backslash V}\left(\boldsymbol{m}^{\prime}\right)$ (which is always 1 for percolation), we obtain

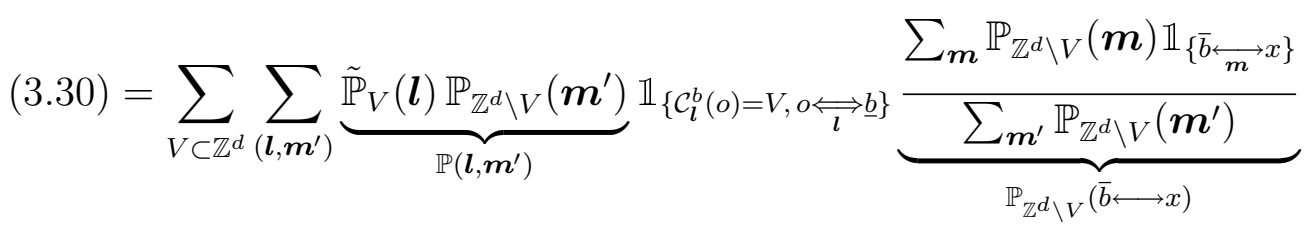

$$
\begin{aligned}
& =\sum_{V \subset \mathbb{Z}^{d}} \mathbb{E}\left[\mathbb{1}_{\left\{\mathcal{C}^{b}(o)=V, o \Longleftrightarrow \underline{b}\right\}} \mathbb{P}_{\mathbb{Z}^{d} \backslash V}(\bar{b} \longleftrightarrow x)\right]=\mathbb{E}\left[\mathbb{1}_{\{o \Longleftrightarrow \underline{b}\}} \mathbb{P}_{\mathbb{Z}^{d} \backslash \mathcal{C}^{b}(o)}(\bar{b} \longleftrightarrow x)\right]
\end{aligned}
$$

Of course, the above derivation using $\mathbb{P}(\boldsymbol{l})$ for $\boldsymbol{l} \in\{0,1\}^{\mathbb{B}_{\mathbb{Z}^{d}}}$ is formal and rather blunt. However, in Section 3.3, we will find similarity in the derivation of an Ising version of this identity.

Finally, by substituting (3.32) back to (3.29), we arrive at

$$
G_{p}^{\mathrm{perc}}(x)=\Delta_{p ; 0}^{\mathrm{perc}}(x)+\sum_{b} \Delta_{p ; 0}^{\mathrm{perc}}(\underline{b}) p_{b} G_{p}^{\mathrm{perc}}(\bar{b}, x)-R_{p ; 1}^{\mathrm{perc}}(x)
$$

where

$$
R_{p ; 1}^{\text {perc }}(x)=\sum_{b} \mathbb{E}\left[\mathbb{1}_{\{o \Longleftrightarrow \underline{b}\}} p_{b}\left(\mathbb{P}(\bar{b} \longleftrightarrow x)-\mathbb{P}_{\mathbb{Z}^{d} \backslash \mathcal{C}^{b}(o)}(\bar{b} \longleftrightarrow x)\right)\right]
$$

This completes the first stage of the expansion.

At every stage of the further expansion, we have to investigate the difference between the full two-point function $G_{p}^{\text {perc }}(y, x) \equiv \mathbb{P}(y \longleftrightarrow x)$ and its restricted version $\mathbb{P}_{\mathbb{Z}^{d} \backslash V}(y \longleftrightarrow$ $x$ ) for given $y \in \mathbb{Z}^{d}$ and $V \subset \mathbb{Z}^{d}$. However, since $\mathbb{P}_{\mathbb{Z}^{d} \backslash V}(y \longleftrightarrow x)=\mathbb{P}\left(y \longleftrightarrow x\right.$ in $\left.\mathbb{Z}^{d} \backslash V\right)$, the difference is actually equal to the probability of the event that $y$ is connected to $x$ in $\mathbb{Z}^{d}$, but not in $\mathbb{Z}^{d} \backslash V$, i.e., every connection from $y$ to $x$ has to go through a vertex in $V$. The key idea to derive the higher-order expansion coefficients is to see when for the first time the connection goes though $V$. If there are no pivotal bonds for that connection after going through $V$, this will be the contribution to $\Delta_{p ; N}^{\text {perc }}(x)$ at the $N^{\text {th }}$ stage of the expansion. On the other hand, if there are pivotal bonds for that connection after going through $V$, then we cut the structure at the first bond among those pivotal bonds, yielding $\left(\Delta_{p ; N}^{\text {perc }} * p D * G_{p}^{\text {perc }}\right)(x)-R_{p ; N+1}^{\text {perc }}(x)$, where the correction term $R_{p ; N+1}^{\text {perc }}(x)$ again contains the difference between a full two-point function and its restricted version. Therefore, we can repeat the same procedure and continue expanding indefinitely. See [16] for more details.

\subsection{Expansion for the Ising model}

In this section, we explain the derivation of the expansion (3.15) for the finite-volume Ising two-point function $\left\langle\varphi_{o} \varphi_{x}\right\rangle_{\Lambda}:=\left\langle\varphi_{o} \varphi_{x}\right\rangle_{p ; \Lambda}$ for $x, o \in \Lambda \subset \mathbb{Z}^{d}$ :

$$
\left\langle\varphi_{o} \varphi_{x}\right\rangle_{\Lambda}=\Delta_{p, \Lambda}^{\mathrm{Ising}}(x)+\sum_{b \in \mathbb{B}_{\Lambda}} \Delta_{p, \Lambda}^{\mathrm{Ising}}(\underline{b}) p_{b}\left\langle\varphi_{\bar{b}} \varphi_{x}\right\rangle_{\Lambda}
$$


To obtain this identity, we use the so-called random-current representation, initiated by Griffiths, Hurst and Sherman [12]. As explained below, it allows us to represent $\left\langle\varphi_{o} \varphi_{x}\right\rangle_{\Lambda}$ as a sort of percolation two-point function. Therefore, we can apply the inclusion-exclusion argument using pivotal bonds, as explained in the previous section. Moreover, the event defining the $N^{\text {th }}$-order expansion coefficient $\Delta_{p, \Lambda ; N}^{\text {Ising }}(x)$ is identical to that for $\Delta_{p ; N}^{\text {perc }}(x)$, for every $N \geq 0$. Then, why is $d_{\mathrm{c}}^{\text {Ising }}$ equal to $d_{\mathrm{c}}^{\mathrm{SAw}}$, not equal to $d_{\mathrm{c}}^{\text {perc }}$, as in $(2.3)-(2.4)$ ? We will get back to this issue at the end of this section.

Now, we explain the random-current representation of $\left\langle\varphi_{x} \varphi_{y}\right\rangle_{\Lambda}$. We call $\boldsymbol{n} \equiv\left\{n_{b}\right\} \in$ $\mathbb{Z}_{+}^{\mathbb{B}_{\Lambda}}$ a current configuration on $\mathbb{B}_{\Lambda}$ (cf., a bond configuration $\boldsymbol{n} \in\{0,1\}^{\mathbb{B}_{\Lambda}}$ for percolation). A vertex $v$ is said to be a source of a current configuration $\boldsymbol{n}$ if $\sum_{b \ni v} n_{b}$ is an odd number, and we denote by $\partial \boldsymbol{n}$ the set of all sources of $\boldsymbol{n}$. Let

$$
W_{\Lambda}(\boldsymbol{n})=\prod_{b \in \mathbb{B}_{\Lambda}} \frac{\left(\beta J_{b}\right)^{n_{b}}}{n_{b} !} .
$$

Then, $\left\langle\varphi_{x} \varphi_{y}\right\rangle_{\Lambda}$ can be represented as (e.g., [12])

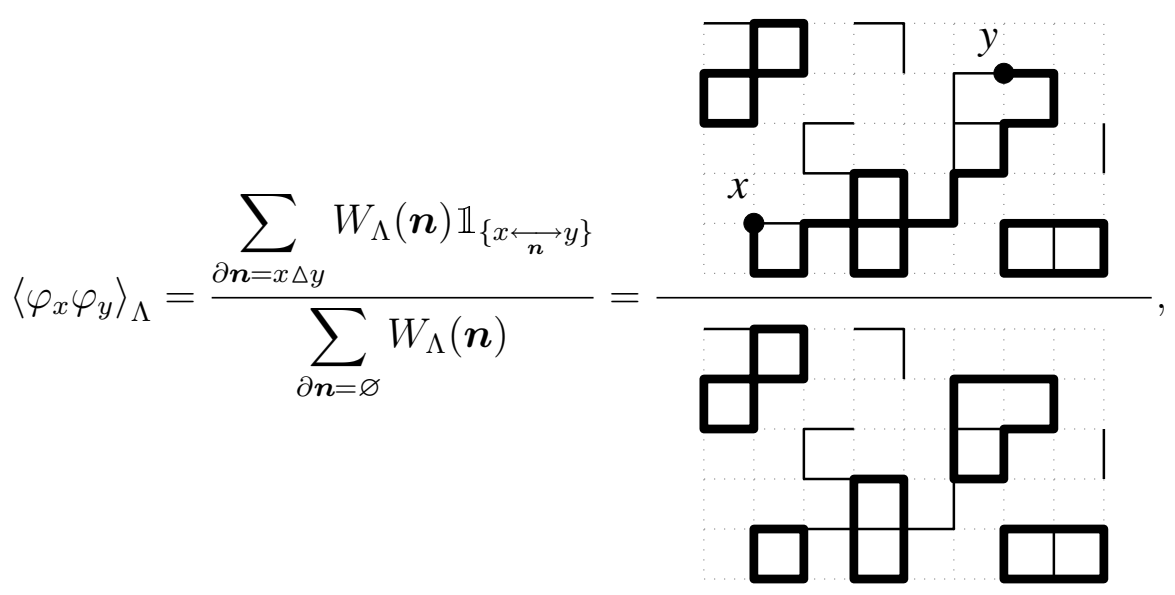

where $x \Delta y$ is an abbreviation for the symmetric difference $\{x\} \triangle\{y\}: x \Delta y=\{x, y\}$ if $x \neq y$, and $x \Delta y=\varnothing$ if $x=y$. In the above figures, each bold line-segment represents a bond with an odd current, while a thinner line-segment represents a bond with a positiveeven current. Although the indicator function in the numerator is redundant because of the source constraint, we keep it here to emphasize that $x$ is $\boldsymbol{n}$-connected to $y$. In fact, $x$ is connected to $y$ with bonds having odd currents. Using this fact, we have, for example,

$$
\begin{aligned}
\sum_{\partial \boldsymbol{n}=x \Delta y} W_{\Lambda}(\boldsymbol{n}) \mathbb{1}_{\{x \overleftrightarrow{n} y\}} & \leq \sum_{v \in \Lambda} \frac{\sum_{n_{x, v} \text { :odd }}\left(\beta J_{x, v}\right)^{n_{x, v}} / n_{x, v} !}{\sum_{m_{x, v} \text { :even }}\left(\beta J_{x, v}\right)^{m_{x, v} / m_{x, v} !}} \sum_{\substack{\partial \boldsymbol{m}=v \Delta y \\
\left(m_{x, v} \text { :even }\right)}} W_{\Lambda}(\boldsymbol{m}) \mathbb{1}_{\{v \overleftrightarrow{m} y\}} \\
& \leq \sum_{v \in \Lambda} \underbrace{\tanh \left(\beta J_{x, v}\right)}_{p_{x, v} \equiv p D(v-x)}\left\langle\varphi_{v} \varphi_{y}\right\rangle_{\Lambda} \sum_{\partial \boldsymbol{n}=\varnothing} W_{\Lambda}(\boldsymbol{n})
\end{aligned}
$$

which implies in the infinite-volume limit (cf., (3.3) and (3.19))

$$
\tilde{G}_{p}^{\text {Ising }}(x, y):=G_{p}^{\text {Ising }}(x, y)-\delta_{x, y} \leq\left(p D * G_{p}^{\text {Ising }}\right)(x, y) .
$$

Repeated use of this inequality yields $G_{p}^{\text {Ising }}(x) \leq S_{p}(x)$ for any $p<1$. 
Derivation of the lace expansion for $\left\langle\varphi_{o} \varphi_{x}\right\rangle_{\Lambda}$. We only explain here the first stage of the derivation of the expansion (3.35), and refer to [25] for the full expansion.

First, we define

$$
\Delta_{p, \Lambda ; 0}^{\mathrm{Ising}}(x)=\frac{\sum_{\partial \boldsymbol{n}=o \Delta x} W_{\Lambda}(\boldsymbol{n}) \mathbb{1}_{\{o \overleftrightarrow{n} x\}}}{\sum_{\partial \boldsymbol{n}=\varnothing} W_{\Lambda}(\boldsymbol{n})}
$$

Then, by (3.27) and (3.37), we obtain

$$
\left\langle\varphi_{o} \varphi_{x}\right\rangle_{\Lambda}=\Delta_{p, \Lambda ; 0}^{\mathrm{Ising}}(x)+\sum_{b \in \mathbb{B}_{\Lambda}} \frac{\sum_{\partial \boldsymbol{n = o \Delta x}} W_{\Lambda}(\boldsymbol{n}) \mathbb{1}_{\{o \overleftrightarrow{\boldsymbol{n}} \underline{b} \text { without using } b\}} \mathbb{1}_{\left\{n_{b}: \text { odd }\right\}} \mathbb{1}_{\left\{\bar{b} \overleftrightarrow{\boldsymbol{n}} x \text { in } \Lambda \backslash \mathcal{C}_{\boldsymbol{n}}^{b}(o)\right\}}}{\sum_{\partial \boldsymbol{n}=\varnothing} W_{\Lambda}(\boldsymbol{n})}
$$

where we have used the fact that $n_{b}>0$ is an odd number if $o \underset{n}{\longleftrightarrow} x$ and $b \in \operatorname{piv}_{\boldsymbol{n}}(o, x)$.

Next, we investigate the numerator of the second term. First, by changing the parity of $n_{b}$ as in (3.38), we obtain

$$
\begin{aligned}
& \sum_{\partial \boldsymbol{n}=o \Delta x} W_{\Lambda}(\boldsymbol{n}) \mathbb{1}_{\{o \overleftrightarrow{\underline{n}} \underline{b} \text { without using } b\}} \mathbb{1}_{\left\{n_{b}: \text { odd }\right\}} \mathbb{1}_{\left\{\bar{b} \overleftrightarrow{n} x \text { in } \Lambda \backslash \mathcal{C}_{\boldsymbol{n}}^{b}(o)\right\}}
\end{aligned}
$$

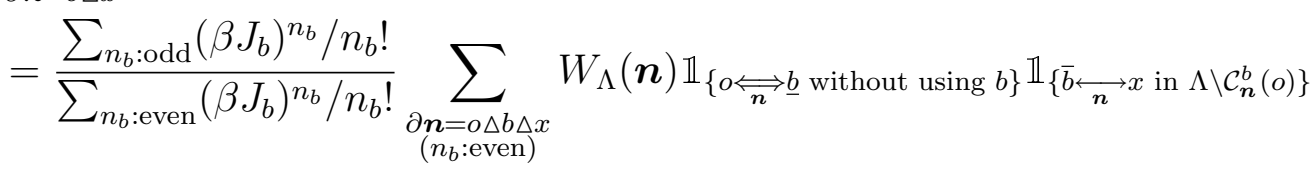

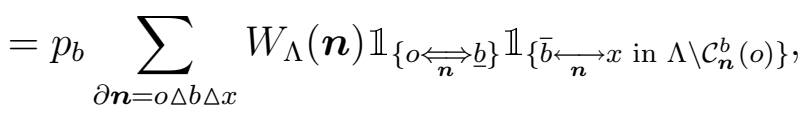

where we have ignored the "without using $b$ " condition, due to the same reason as explained below (3.29); we have also dropped the " $n_{b}$ :even" condition, because it is impossible to satisfy the source constraint and the conditions in the indicators at the same time if $n_{b}$ is an odd number. By conditioning on the cluster $\mathcal{C}^{b}(o)$, we have (cf., (3.30))

$$
\begin{aligned}
& (3.42)=p_{b} \sum_{V \subset \Lambda} \sum_{\partial \boldsymbol{n}=o \Delta b \Delta x} W_{\Lambda}(\boldsymbol{n}) \mathbb{1}_{\left\{\mathcal{C}_{\boldsymbol{n}}^{b}(o)=V, o \overleftrightarrow{\boldsymbol{n}} \underline{b}\right\}} \mathbb{1}_{\left\{\bar{b} \overleftrightarrow{{ }_{n}} x \text { in } \Lambda \backslash V\right\}}
\end{aligned}
$$

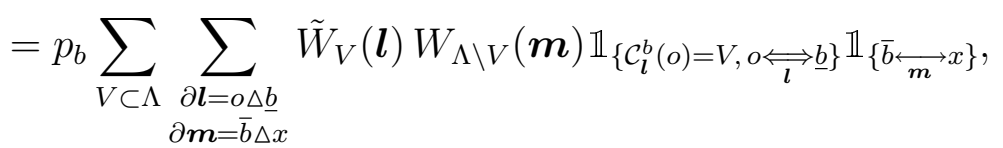

where $\boldsymbol{n} \equiv(\boldsymbol{l}, \boldsymbol{m})$ is a current configuration on $\mathbb{B}_{\Lambda}$, with $\boldsymbol{m} \in \mathbb{Z}_{+}^{\mathbb{B}_{\Lambda \backslash V}}$ and $\boldsymbol{l} \in \mathbb{Z}_{+}^{\mathbb{B}_{\Lambda} \backslash \mathbb{B}_{\Lambda \backslash V}}$, and $\tilde{W}_{V}$ is such that $W_{\Lambda}(\boldsymbol{l}, \boldsymbol{m})=\tilde{W}_{V}(\boldsymbol{l}) W_{\Lambda \backslash V}(\boldsymbol{m})$. Here, in order for a joint current configuration $(\boldsymbol{l}, \boldsymbol{m})$ to satisfy all the conditions in the first line, $\boldsymbol{l}$ and $\boldsymbol{m}$ have to satisfy the required source constraints in the second line. Inspired by the computation in (3.32), we multiply and divide (3.43) by $\sum_{\partial \boldsymbol{m}^{\prime}=\varnothing} W_{\Lambda \backslash V}\left(\boldsymbol{m}^{\prime}\right)$ (which is not 1 in general for the 
Ising model) and obtain

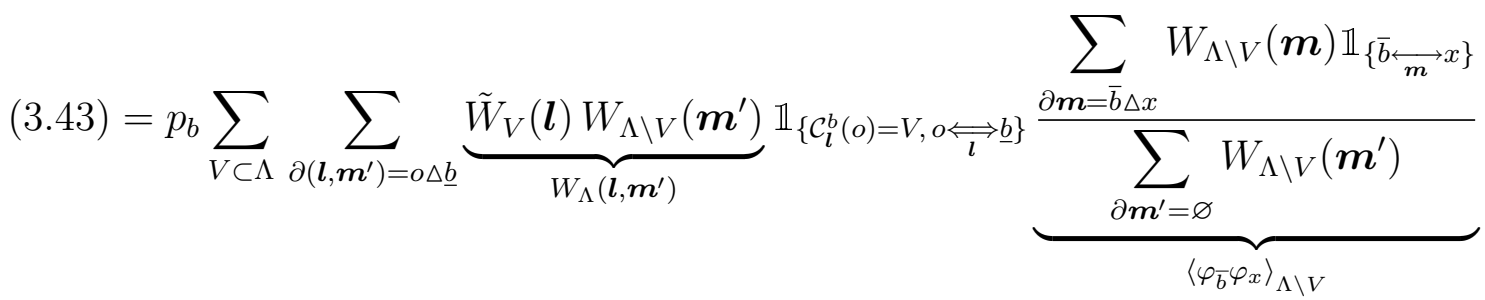

$$
\begin{aligned}
& =\sum_{\partial \boldsymbol{n}=o \Delta \underline{b}} W_{\Lambda}(\boldsymbol{n}) \mathbb{1}_{\{o \overleftrightarrow{n} \underline{b}\}} p_{b}\left\langle\varphi_{\bar{b}} \varphi_{x}\right\rangle_{\Lambda \backslash \mathcal{C}_{\boldsymbol{n}}^{b}(o)} .
\end{aligned}
$$

Finally, by substituting (3.44) back to (3.41), we arrive at

$$
\left\langle\varphi_{o} \varphi_{x}\right\rangle_{\Lambda}=\Delta_{p, \Lambda ; 0}^{\text {Ising }}(x)+\sum_{b \in \mathbb{B}_{\Lambda}} \Delta_{p, \Lambda ; 0}^{\text {Ising }}(\underline{b}) p_{b}\left\langle\varphi_{\bar{b}} \varphi_{x}\right\rangle_{\Lambda}-R_{p, \Lambda ; 1}^{\text {Ising }}(x),
$$

where

$$
R_{p, \Lambda ; 1}^{\text {Ising }}(x)=\sum_{b \in \mathbb{B}_{\Lambda}} \frac{\sum_{\partial \boldsymbol{n}=o \Delta \underline{b}} W_{\Lambda}(\boldsymbol{n}) \mathbb{1}_{\{o \overleftrightarrow{n} \underline{b}\}} p_{b}\left(\left\langle\varphi_{\bar{b}} \varphi_{x}\right\rangle_{\Lambda}-\left\langle\varphi_{\bar{b}} \varphi_{x}\right\rangle_{\Lambda \backslash \mathcal{C}_{n}^{b}(o)}\right)}{\sum_{\partial \boldsymbol{n}=\varnothing} W_{\Lambda}(\boldsymbol{n})} .
$$

This completes the first stage of the expansion.

Similarly to percolation, at every stage of the further expansion, we have to investigate $\left\langle\varphi_{y} \varphi_{x}\right\rangle_{\Lambda}-\left\langle\varphi_{y} \varphi_{x}\right\rangle_{\Lambda \backslash V}$ for given $y \in \Lambda$ and $V \subset \Lambda$. However, since $\sum_{\partial \boldsymbol{n}=\varnothing} W_{A}(\boldsymbol{n}) \neq 1$ for any nontrivial $A \subset \mathbb{Z}^{d}$, we have

$$
\left\langle\varphi_{y} \varphi_{x}\right\rangle_{\Lambda \backslash V} \neq \frac{\sum_{\partial \boldsymbol{n = y \Delta x}} W_{\Lambda}(\boldsymbol{n}) \mathbb{1}_{\{y \overleftrightarrow{n} x \text { in } \Lambda \backslash V\}}}{\sum_{\partial \boldsymbol{n}=\varnothing} W_{\Lambda}(\boldsymbol{n})},
$$

in contrast with the equality $\mathbb{P}_{\Lambda \backslash V}(y \longleftrightarrow x)=\mathbb{P}(y \longleftrightarrow x$ in $\Lambda \backslash V)$ for percolation. This makes it difficult to compare two-point functions on different sets of sites.

In [25], we overcame this difficulty using the so-called source-switching lemma [12] and proved that

$$
\left\langle\varphi_{y} \varphi_{x}\right\rangle_{\Lambda}-\left\langle\varphi_{y} \varphi_{x}\right\rangle_{\Lambda \backslash V}=\frac{\sum_{\substack{\partial \boldsymbol{n}=y \Delta x \\ \partial \boldsymbol{m}=\varnothing}} W_{\Lambda}(\boldsymbol{n}) W_{\Lambda \backslash V}(\boldsymbol{m}) \mathbb{1}_{\{y \overleftrightarrow{\boldsymbol{m}+\boldsymbol{n}} x \text { through } V\}}}{\sum_{\partial \boldsymbol{n}=\partial \boldsymbol{m}=\varnothing} W_{\Lambda}(\boldsymbol{n}) W_{\Lambda \backslash V}(\boldsymbol{m})},
$$

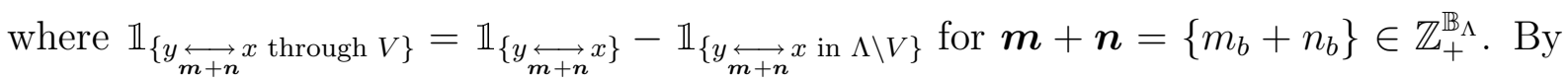
this percolation representation, we can follow the same strategy as for percolation: find the first bond $b \in \operatorname{piv}_{\boldsymbol{m}+\boldsymbol{n}}(y, x)$ such that $x \underset{\boldsymbol{m + n}}{\overleftrightarrow{b}}$ though $V$. At the $N^{\text {th }}$ stage of the expansion, the contribution from the case in which there are no such pivotal bonds is $\Delta_{p, \Lambda ; N}^{\text {Ising }}(x)$, otherwise we have $\sum_{b} \Delta_{p, \Lambda ; N}^{\text {Ising }}(\underline{b}) p_{b}\left\langle\varphi_{\bar{b}} \varphi_{x}\right\rangle_{\Lambda}-R_{p, \Lambda ; N+1}^{\text {Ising }}(x)$, where the correction term $R_{p, \Lambda ; N+1}^{\text {Ising }}(x)$ again contains the difference of the form (3.48). Then, we can repeat the same argument to continue the expansion indefinitely. See [25] for more details. 
Remark: Since we have exploited the percolation structure of the random-current representation, the set of "bond" configurations defining $\Delta_{p, \Lambda ; N}^{\text {Ising }}(x)$ is identical to that for $\Delta_{p ; N}^{\text {perc }}(x)$, for every $N \geq 0$. As explained in Section 3.2, in order to bound $\Delta_{p ; N}^{\text {perc }}(x)$ for percolation, it suffices to know the geometrical structure of the relevant bond configurations. Then, the BK inequality does the remaining job: e.g., $\Delta_{p ; 0}^{\text {perc }}(x) \leq G_{p}^{\text {perc }}(x)^{2}$. However, if this was also the case for the Ising model, i.e., if there was an Ising version of the BK inequality such that $\Delta_{p, \Lambda ; 0}^{\text {Ising }}(x) \leq G_{p}^{\text {Ising }}(x)^{2}$, then $d_{\mathrm{c}}^{\text {Ising }}$ would be equal to $d_{\mathrm{c}}^{\text {perc }}$ (above which the triangle condition holds) rather than $d_{\mathrm{c}}^{\mathrm{SAW}}$ (above which the bubble condition holds, as required for the mean-field behavior for the Ising model).

In fact, instead of using visible clusters of positive-current bonds, we should use a graph $\mathbb{G}_{\Lambda}(\boldsymbol{n}) \equiv\left(\Lambda, E_{\Lambda}(\boldsymbol{n})\right)$ that is constructed from each current configuration $\boldsymbol{n} \in \mathbb{Z}_{+}^{\mathbb{B}_{\Lambda}}$ by joining $u, v \in \Lambda$ with $n_{u, v}$ labeled edges. Then, for each $\boldsymbol{n} \in \mathbb{Z}_{+}^{\mathbb{B}_{\Lambda}}$ defining $\Delta_{p, \Lambda ; 0}^{\text {Ising }}(x)$, there are at least three edge-disjoint paths from $o$ to $x$ in the graph $\mathbb{G}_{\Lambda}(\boldsymbol{n})$, as shown in the following example:

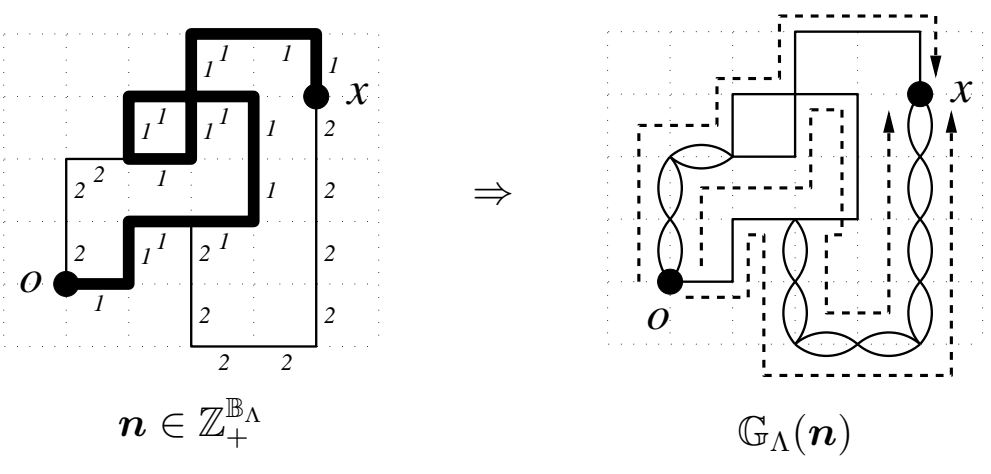

This observation leads us to predict that $\Delta_{p, \Lambda ; 0}^{\text {Ising }}(x)$ is bounded similarly to $\Delta_{p ; 1}^{\mathrm{SAW}}(x)$. The prediction was proved affirmative in $[25]: \Delta_{p, \Lambda ; 0}^{\text {Ising }}(x)-\delta_{x, o} \leq \tilde{G}_{p}^{\text {Ising }}(x)^{3}$. It was also proved that the higher-order expansion coefficients for the Ising model obey similar diagrammatic bounds for the SAW expansion coefficients. This is why $d_{\mathrm{c}}^{\text {Ising }}=d_{\mathrm{c}}^{\mathrm{SAW}}$, not $d_{\mathrm{c}}^{\text {Ising }}=d_{\mathrm{c}}^{\text {perc }}$.

\section{Acknowledgements}

The analysis of the lace expansion explained in this article is taken out of the recent joint work with Lung-Chi Chen [10] and with Markus Heydenreich and Remco van der Hofstad [18]. I am grateful to them for the fruitful and enjoyable collaboration. I would also like to express my thanks to the Institute of Mathematics at Academia Sinica, the London Mathematical Society and the Netherlands Organisation for Scientific Research (NWO) for supporting the travel expenses.

\section{References}

[1] M. Aizenman. Geometric analysis of $\phi^{4}$ fields and Ising models. Comm. Math. Phys., 86:1-48, 1982.

[2] M. Aizenman and D.J. Barsky. Sharpness of the phase transition in percolation models. Comm. Math. Phys., 108:489-526, 1987. 
[3] M. Aizenman, D.J. Barsky, and R. Fernández. The phase transition in a general class of Ising-type models is sharp. J. Stat. Phys., 47:343-374, 1987.

[4] M. Aizenman and C.M. Newman. Tree graph inequalities and critical behavior in percolation models. J. Stat. Phys., 36:107-143, 1984.

[5] J. van den Berg and H. Kesten. Inequalities with applications to percolation and reliability. J. Appl. Probab., 22:556-569, 1985.

[6] T. Bodineau. Translation invariant Gibbs states for the Ising model. Probab. Theory Related Fields, 135:153-168, 2006.

[7] C. Borgs, J.T. Chayes, R. van der Hofstad, G. Slade, and J. Spencer. Random subgraphs of finite graphs. II. The lace expansion and the triangle condition. Ann. Probab., 33:1886-1944, 2005.

[8] D. Brydges and T. Spencer. Self-avoiding walk in 5 or more dimensions. Comm. Math. Phys., 97:125-148, 1985.

[9] F. Camia and C.M. Newman. Critical percolation exploration path and $S L E_{6}$ : a proof of convergence. Probab. Theory Related Fields, 139:473-519, 2007.

[10] L.-C. Chen and A. Sakai. Critical behavior for long-range oriented percolation. I. Probab. Theory Related Fields, in press.

[11] R. Fernández, J. Fröhlich, and A.D. Sokal. Random Walks, Critical Phenomena, and Triviality in Quantum Field Theory. Springer, Berlin, 1992.

[12] R.B. Griffiths, C.A. Hurst, and S. Sherman. Concavity of magnetization of an Ising ferromagnet in a positive external field. J. Math. Phys., 11:790-795, 1970.

[13] G. Grimmett. Percolation (2nd edition). Springer, Berlin, 1999.

[14] T. Hara. Decay of correlations in nearest-neighbour self-avoiding walk, percolation, lattice trees and animals. Ann. Probab., 36:530-593, 2008.

[15] T. Hara, R. van der Hofstad, and G. Slade. Critical two-point functions and the lace expansion for spread-out high-dimensional percolation and related models. Ann. Probab., 31:349-408, 2003.

[16] T. Hara and G. Slade. Mean-field critical behaviour for percolation in high dimensions. Comm. Math. Phys., 128:333-391, 1990.

[17] T. Hara and G. Slade. Self-avoiding walk in five or more dimensions. I. The critical behaviour. Comm. Math. Phys., 147:101-136, 1992.

[18] M. Heydenreich, R. van der Hofstad, and A. Sakai. Mean-field behavior for long- and finite-range Ising model, percolation and self-avoiding walk. Preprint, 2008.

[19] R. van der Hofstad and M. Holmes. An expansion for self-interacting random walks. Preprint, 2006. 
[20] R. van der Hofstad and A. Sakai. Critical points for spread-out self-avoiding walk, percolation and the contact process above the upper critical dimensions. Probab. Theory Related Fields, 132:438-470, 2005.

[21] R. van der Hofstad and G. Slade. A generalised inductive approach to the lace expansion. Probab. Theory Related Fields, 122:389-430, 2002.

[22] M. Holmes and A. Sakai. Senile reinforced random walks. Stochastic Process. Appl., 117:1519-1539, 2007.

[23] E.H. Lieb. A refinement of Simon's correlation inequality. Comm. Math. Phys., $77: 127-135,1980$.

[24] N. Madras and G. Slade. The Self-Avoiding Walk. Birkhäuser, Boston, 1993.

[25] A. Sakai. Lace expansion for the Ising model. Comm. Math. Phys., 272:283-344, 2007.

[26] B. Simon. Correlation inequalities and the decay of correlations in ferromagnets. Comm. Math. Phys., 77:111-126, 1980.

[27] G. Slade. The lace expansion and its applications. Lecture Notes in Math., 1879, 2006.

[28] S. Smirnov and W. Werner. Critical exponents for two-dimensional percolation. Math. Res. Lett., 8:729-744, 2001.

[29] W. Werner. Random planer curves and Schramm-Loewner evolutions. Lecture Notes in Math., 1840:107-195, 2004. 